\title{
Catheter-related deep vein thrombosis in newborn infants
}

\author{
María C. Rubio Longo, M.D. ${ }^{a}$, Patricio M. De Lucca, M.D. ${ }^{a}$, Gustavo Goldsmit, M.D. ${ }^{a}$,
} Diana Fariña, M.D. ${ }^{a}$, José Lipsich, M.D. ${ }^{b}$ and Susana Rodríguez, M.D. ${ }^{c}$

\begin{abstract}
Introduction. In neonatology, more than $90 \%$ of deep vein thromboses are related to catheter use. The objective of this study was to determine the incidence and risk factors associated with central venous catheter-related deep vein thrombosis. Population and methods. Observational and analytical study conducted in a prospective cohort. All central venous catheters were included using consecutive, non-random sampling. The screening protocol included a daily clinical examination and a Doppler ultrasound (7-10 days after insertion and/or $72 \mathrm{~h}$ after removal). Follow-up: from catheter insertion to catheter removal, death or patient counter-referral. The incidence density rate per 1000 catheter-days was estimated with its corresponding $95 \%$ confidence intervals (CIs), and risk factors, using Cox multivariate analysis. Results. Thrombosis was identified in 22/264 catheters. The incidence density rate of thrombosis was $5.33 \%$ o catheter-days (95\% CI: 3.34-8.07). Cardiovascular surgery and and the use of central catheters others than peripherally inserted ones, were independent risk factors for thrombosis (hazard ratio: 3.8 [95\% CI: 1.6-9] and 2.75 [95\% CI: 1.17-6.45]).
\end{abstract}

Conclusions. The incidence of central venous catheter-related deep vein thrombosis was 5.33 per 1000 catheter-days. A history of cardiovascular surgical procedures and and the use of central catheters others than peripherally inserted ones, were associated with a higher risk of this complication.

Key words: thrombosis, newborn infant, vascular access devices, risk factors.

http: / / dx.doi.org/10.5546/ aap.2021.eng.32

To cite: Rubio Longo MC, De Lucca PM, Goldsmit G, Fariña D, etal. Catheter-related deep vein thrombosis in newborn infants. Arch Argent Pediatr 2021;119(1):32-38.

E-mail address:

María C. Rubio Longo,

M.D.:

crubio@garrahan.gov.ar

Funding:

Grant awarded by

Fundación Hospital

Garrahan.

Conflict of interest:

None.

Received: 2-14-2020

Accepted: 7-30-2020

\section{GLOSSARY}

CVC: central venous catheter.

DVT: deep vein thrombosis.

NICU: neonatal intensive care unit.

\section{INTRODUCTION}

Deep vein thrombosis (DVT) is one of the most common complications related to central venous catheter (CVC) use in the neonatal intensive care unit (NICU). It is associated with short-term difficulties, such as local obstruction and the formation of emboli, and medium- to long-term difficulties, such as lack of thrombus resolution and the development of post-thrombotic syndrome in more than one third of patients. ${ }^{1,2}$

The Neonatal Intensive Care Unit of Hospital Garrahan is a level IIIb unit that receives approximately 600 newborn infants every year. A high percentage of these patients are critically ill and require highly complex procedures or treatments. Such a critical level requires using a safe venous access, sometimes for a long time, which is why it is common to place one or more CVCs during hospitalization.

Previous data from a retrospective cohort with patients requiring lowmolecular-weight heparin treatment at our unit showed an incidence of catheter-related DVT of $3.4 \%$ and $2.9 \%$ in 2013 and 2014, respectively. In some of these patients, DVT was diagnosed while performing an ultrasound for another reason, with no evidence of clinical signs of venous occlusion. These data show that the incidence of catheter-related DVT in our population may depend on the diagnostic method used and, therefore, a systematic ultrasound screening in children with CVC may alter it. 
The objective of this study was to establish prospectively the incidence and risk factors of CVC-related DVT in a cohort of patients admitted to a level IIIb NICU under a systematic protocol of clinical and ultrasound diagnosis.

\section{POPULATION AND METHODS}

This was an observational and analytical study conducted in a prospective cohort. All CVCs (unit of analysis) inserted between August 2015 and July 2016 at the NICU of Hospital Garrahan were included using consecutive, non-random sampling. Peripherally inserted catheters (made of silicone rubber), short- and long-term central catheters (both made of polyurethane), and umbilical venous catheters (made of polyurethane) were eligible. Catheters inserted outside of our hospital and/or in children undergoing anticoagulant therapy were excluded.

All included catheters were checked following a uniform protocol, which included a daily clinical examination and a Doppler ultrasound 7-10 days after its insertion and/or $72 \mathrm{~h}$ after its removal. This allowed to perform between 1 and 2 follow-up ultrasound examinations for each catheter, depending on whether it was removed before/after 7-10 days. In addition, the primary care physician could also ask for an ultrasound in the presence of signs and/or symptoms compatible with DVT outside of the above-mentioned time frames.

DVT, the outcome variable in this study, was defined by the presence of the following ultrasound criteria: echogenic material inside the vessel, lack of color flow in the Doppler ultrasound venous mapping, lack of vein compressibility (jugular and femoral veins), increased vein diameter, and/or presence of collateral circulation. When ultrasound results were not conclusive, a second diagnostic test was conducted (phlebography in the presence of doubtful results for limbs and great vessels, or echocardiogram for intracardiac imaging or great vessels adjacent to the heart) based on the diagnostic algorithm used at our hospital.

Tests were performed and recorded by different operators. Nevertheless, to confirm the diagnosis, all of them were reviewed by only one imaging specialist, who was not aware of the relation between the tests and patients' characteristics. DVT was considered symptomatic if any of the following signs and/or symptoms were present: edema and / or discoloration of the limb, superior vena cava syndrome or chylothorax.
The follow-up duration of included catheters lasted from their insertion to their removal, patient death or counter-referral of the patient with the catheter in place to a different facility. During this period, outcome measures related to CVC characteristics were collected: type (peripherally inserted, short-term, long-term, and umbilical), internal diameter in French, number of lumens, insertion site, parenteral nutrition and inotropic use through the catheter, reason for removal, and duration of use; and those related to patients' characteristics: gestational age, birth weight, mode of delivery, maternal history, perinatal asphyxia, sex, underlying condition, need for assisted mechanical ventilation, surgery, extracorporeal membrane oxygenation (ECMO), sepsis, catheter-related infection, previous thrombosis, length of stay, and clinical course. For those catheters related to venous thrombosis, clinical signs, complications, and treatment were recorded.

Data were entered into an Excel spreadsheet and transferred to the Stata 12.0 statistical package for their analysis. They were described using frequency tables and measures of central tendency and dispersion. The incidence of catheter-related thrombosis was expressed as incidence rate and incidence density rate per 1000 catheter-days, with their corresponding $95 \%$ confidence intervals (CIs). The probability of developing a thrombotic event during follow-up was estimated using the Kaplan-Meier method, and associated risk factors were determined using a bivariate analysis with the log-rank test and a multivariate analysis based on Cox proportional hazards model, reporting hazard ratios (HR) and $95 \%$ CIs. Results were considered significant if the value of $p$ was $<0.05$. The study protocol was submitted to and approved by the Research Ethics Review Committee of Hospital Garrahan. An informed consent for study participation was obtained from patients' families.

\section{RESULTS}

During the study period, 507 patients were admitted to the unit; $34 \%(n=171)$ required CVC. A total of 264 catheters were included in the study (31 did not meet eligibility criteria) (Figure 1).

The characteristics of patients with included catheters were the following: $54 \%$ (n: 93) were term newborn infants, with a median gestational age of 37 weeks (range: 24-42); the main reasons for hospitalization were general surgical conditions $(63 \%)$, congenital heart disease $(13 \%)$, and 
other respiratory and infectious diseases $(24 \%)$. General surgery was required in $59 \%$ of cases; cardiovascular surgery, in $8.6 \%$; and both, in $5.7 \%$. A total of $36 \%$ required only one catheter during their hospitalization; and $64 \%$, two or more.

Thrombosis was identified in 22 of 264 catheters, with an incidence rate of $8.33 \%$ (95\% CI: 5.22-12.61). The total risk time was 4127 days, and the incidence density rate of thrombosis, $5.33 \%$ catheter-days (95\% CI: 3.348.07). Figure 2 shows the actuarial survival curve using the Kaplan-Meier method. The events (DVT) were observed to occur as of the first days after insertion; $73 \%$ of thromboses $(16 / 22)$ corresponded to the first 15 days of follow-up.

Of the 22 catheters with DVT, clinical signs were detected in only 3 cases, which is why the incidence rate of symptomatic thrombosis was much lower than that estimated with the ultrasound screening protocol: $1.13 \%$ (95\% CI: $0.23-3.32)$; and the incidence density rate was $0.72 \%$ catheter-days (95\% CI: 0.13-2.12). The 22 thrombotic complications were observed in
20 patients and were treated based on the criteria agreed with the Department of Hematology.

Regarding the characteristics of the 264 studied catheters, peripherally inserted ones were most commonly used (66\%). A total of $32 \%$ had a French size $\geq 4$, and $70 \%$ had $\geq 2$ lumens. As far as the site of insertion, $78 \%$ were placed on the upper half of the body (22 on the right side of the head or neck, 22 on the left side of the head or neck, 72 on the right upper limb, and 90 on the left upper limb), and the remaining $22 \%$, on the lower half of the body (21 on the right lower limb, 19 on the left lower limb, and 18 in the umbilical vein). Out of all catheters, 152 were electively removed due to end of use; 69 , due to complications (22 were mechanical; 28, infectious; and 19, related to misplacement as per follow-up ultrasound); and 19, due to accidental catheter removal. The median indwelling time of catheters with thrombosis was 14 days (interquartile range [IQR]: 4-23) versus 12.5 days (IQR: 6-21) in the case of catheters without thrombosis, and this difference was not significant $(p=0.69)$.

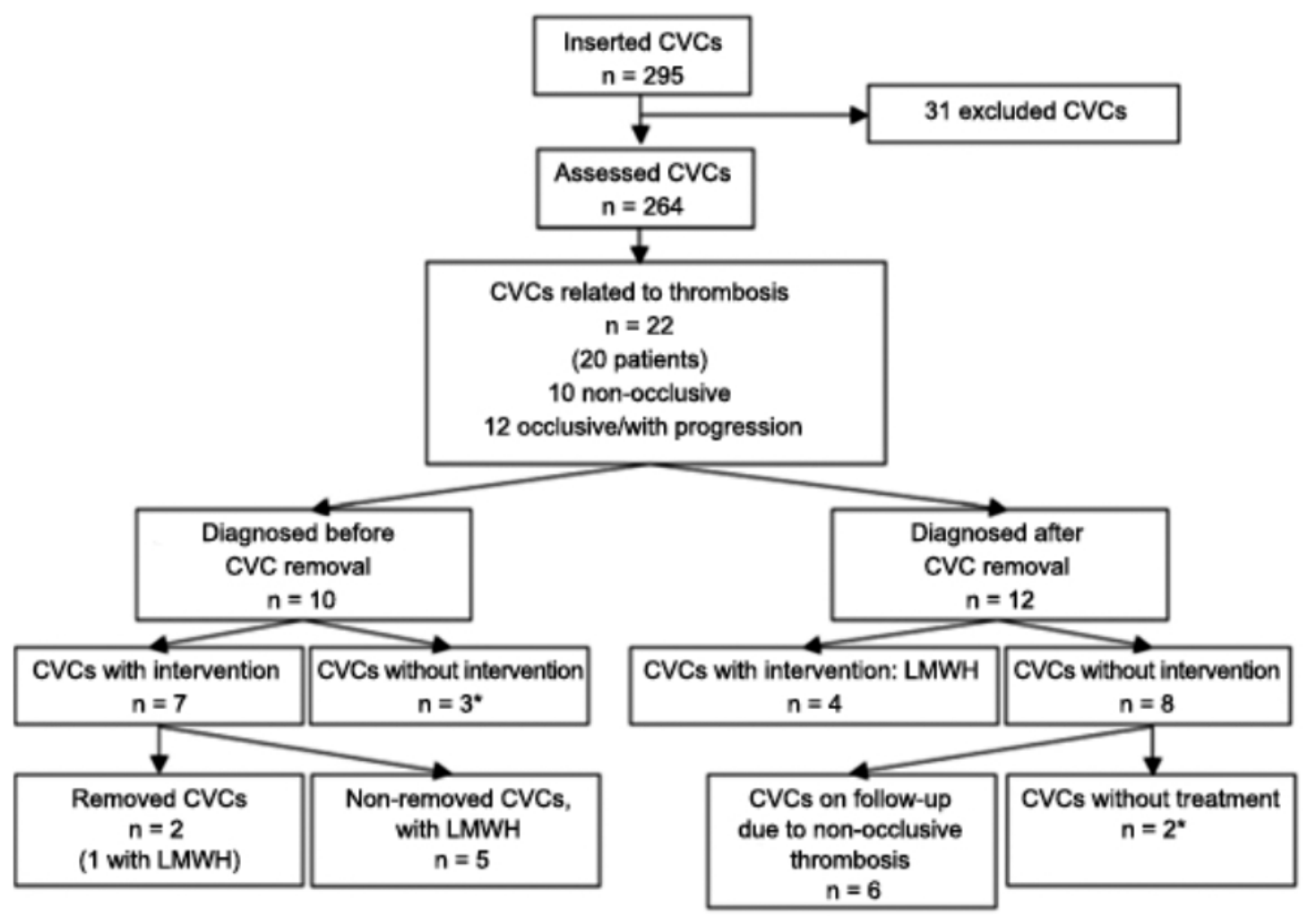

* No intervention due to the patient's critical condition.

${ }^{* *}$ CVC: central venous catheter; LMWH: low-molecular-weight heparin. 
During the study period, 483 ultrasounds were performed, and DVT was identified in 22 cases; 12 of them were occlusive or showed progression on ultrasound follow-up. Of the 22 diagnoses, 10 were detected in the first ultrasound, which was performed before removing the catheter,

FIGURE 2. Cumulative risk of the incidence of central venous catheater-related thrombosis

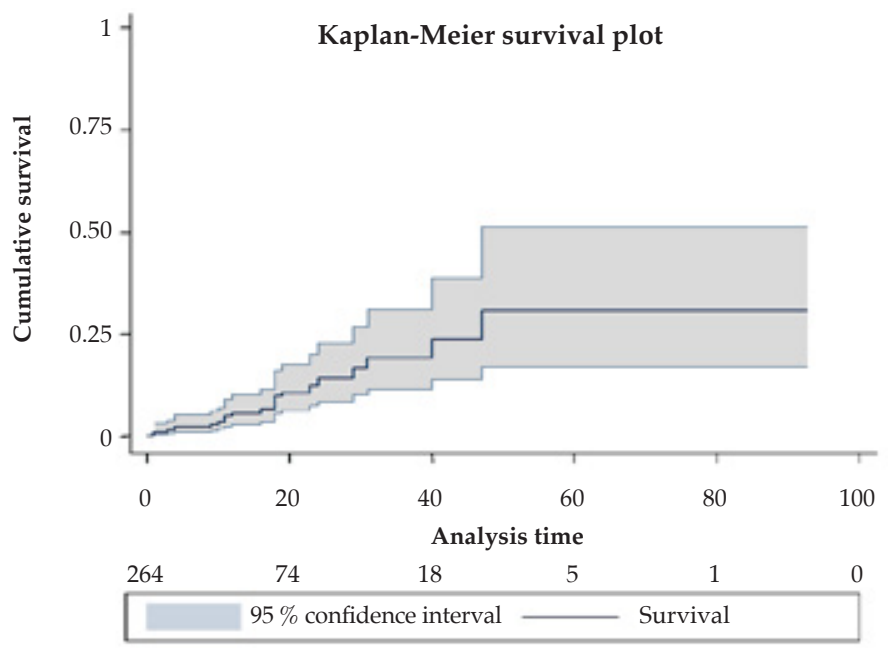

TABLE 1. Central venous catheter-related deep vein thrombosis events

\begin{tabular}{|c|c|c|c|c|c|c|c|}
\hline $\begin{array}{l}\text { Event } \\
n=22\end{array}$ & $\begin{array}{c}\text { Patient } \\
n=20\end{array}$ & Symptoms & $\begin{array}{l}\text { Diagnosed } \\
\text { before } \\
\text { CVC removal } \\
\text { ( } 1^{\text {st }} \text { ultrasound) }\end{array}$ & $\begin{array}{c}\text { Diagnosed } \\
\text { after } \\
\text { CVC removal } \\
\left(2^{\text {nd }} \text { ultrasound }\right)\end{array}$ & $\begin{array}{c}\mathrm{LMWH} \\
\text { treatment }\end{array}$ & $\begin{array}{l}\text { Treatment- } \\
\text { associated } \\
\text { complications }\end{array}$ & $\begin{array}{l}\text { Clinical } \\
\text { course }\end{array}$ \\
\hline 1 & 1 & No & & $X$ & Yes & No & Discharged \\
\hline 2 & 2 & No & $X$ & & Yes & No & $\begin{array}{c}\text { Discharged with } \\
\text { LMWH }\end{array}$ \\
\hline 3 & 3 & No & $X$ & & Yes & Gastrointestinal bleeding & Discharged \\
\hline 4 & & $\begin{array}{l}\text { Superior vena } \\
\text { cava syndrome }\end{array}$ & $x$ & & Yes & Gastrointestinal bleeding & Discharged \\
\hline 5 & 4 & No & & $X$ & No & & Discharged \\
\hline 6 & 5 & No & $X$ & & Yes & Thigh hematoma & Discharged \\
\hline 7 & 6 & No & $X$ & & No & & Discharged \\
\hline 8 & 7 & No & & $X$ & Yes & No & $\begin{array}{c}\text { Discharged with } \\
\text { LMWH }\end{array}$ \\
\hline 9 & 8 & No & & $X$ & No & & Discharged \\
\hline 10 & 9 & No & & $X$ & Yes & No & Death \\
\hline 11 & 10 & No & $X$ & & No & No & Discharged \\
\hline 12 & & No & & $X$ & & & Discharged \\
\hline 13 & 11 & $\begin{array}{l}\text { Local symptoms } \\
\text { in the lower limbs }\end{array}$ & $x$ & & No & & Death \\
\hline 14 & 12 & No & & $x$ & No & & Death \\
\hline 15 & 13 & No & & $X$ & No & & Discharged \\
\hline 16 & 14 & No & & $X$ & Yes & No & $\begin{array}{c}\text { Discharged with } \\
\text { LMWH }\end{array}$ \\
\hline 17 & 15 & No & & $X$ & No & & Discharged \\
\hline 18 & 16 & No & $X$ & & No & & Discharged \\
\hline 19 & 17 & No & $x$ & & Yes & No & Death \\
\hline 20 & 18 & $\begin{array}{l}\text { Superior vena } \\
\text { cava syndrome }\end{array}$ & $x$ & & Yes & No & $\begin{array}{c}\text { Discharged with } \\
\text { LMWH }\end{array}$ \\
\hline 21 & 19 & No & & $x$ & No & & Discharged \\
\hline 22 & 20 & No & & $X$ & No & & Discharged \\
\hline
\end{tabular}

${ }^{*} \mathrm{X}$ : Time of diagnosis.

${ }^{* *}$ CVC: central venous catheter; LMWH: low-molecular-weight heparin. 
and 12 were diagnosed after its removal. Table 1 shows the details of each case, treatment, and clinical course.

\section{Risk factors}

A history of maternal hypertension, the presence of cardiovascular surgery, the use of catheters other than peripherally inserted ones, catheter insertion in the lower half of the body, inotropic infusion, and a French size above 4 were factors associated with a higher frequency of DVT per 1000 catheter-days. In the multivariate

FIGURE 3. Cumulative risk of central venous catheter-related thrombosis by type of catheter and history of cardiovascular surgery

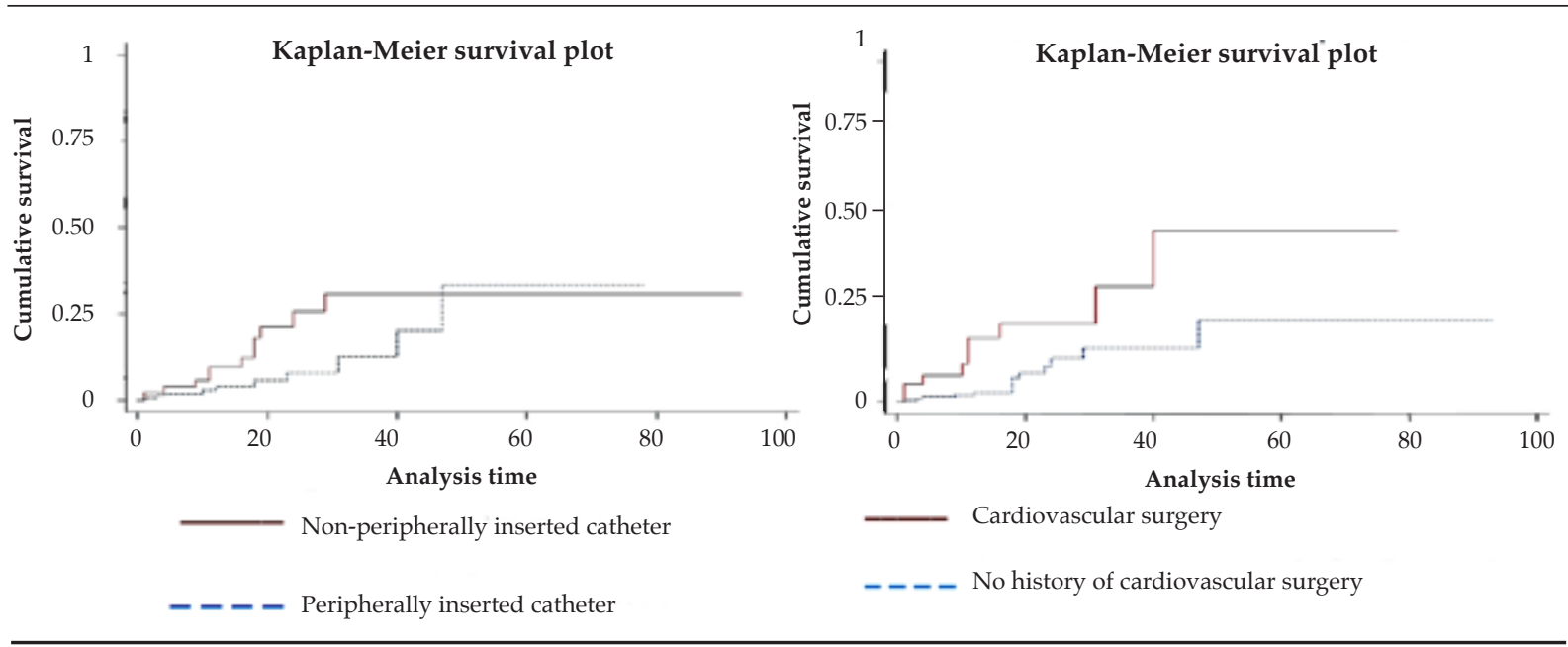

TABLE 2. Risk factors associated with central venous thrombosis. Bivariate and multivariate analysis

\begin{tabular}{|c|c|c|c|c|c|c|}
\hline Factor & $\begin{array}{c}\text { Incidence } \\
\text { density } \\
\text { rate (DVT/1000 } \\
\text { catheter-days) } \\
\text { WITH the } \\
\text { risk factor }\end{array}$ & $\begin{array}{c}\text { Incidence } \\
\text { density } \\
\text { rate (DVT/1000 } \\
\text { catheter-days) } \\
\text { WITHOUT the } \\
\text { risk factor }\end{array}$ & $\begin{array}{c}\text { Unadjusted } \\
\text { HR } \\
(95 \% \mathrm{CI})^{*}\end{array}$ & $p$ value & $\begin{array}{c}\text { Adjusted } \\
\text { HR } \\
(95 \% \mathrm{CI})^{* *}\end{array}$ & $p$ value \\
\hline Maternal hypertension & 21,74 & 4.56 & $\begin{array}{c}4.64 \\
(1.56-3.84)\end{array}$ & $0.006^{*}$ & & \\
\hline History of cardiovascular surgery & 12.77 & 3.80 & $\begin{array}{c}3.32 \\
(1.41-7.80)\end{array}$ & $0.006^{*}$ & $\begin{array}{c}3.8 \\
(1.6-9.03)\end{array}$ & 0.002 \\
\hline Inotropic infusion & 8.50 & 3.22 & $\begin{array}{c}2.59 \\
(1.08-6.18)\end{array}$ & $0.03^{*}$ & & \\
\hline $\begin{array}{l}\text { Catheter insertion in the } \\
\text { lower half of the body }\end{array}$ & 10.71 & 4.3 & $\begin{array}{c}2.53 \\
(1.02-6.26)\end{array}$ & $0.04^{*}$ & & \\
\hline NON-peripherally inserted catheter & 8.75 & 3.62 & $\begin{array}{c}2.37 \\
(1.02-5.5)\end{array}$ & $0.04^{*}$ & $\begin{array}{c}2.75 \\
(1.17-6.45)\end{array}$ & 0.02 \\
\hline Catheter $\geq 4$ French & 8.73 & 3.83 & $\begin{array}{c}2.28 \\
(0.98-5.27)\end{array}$ & 0.055 & & \\
\hline ECMO & 15.70 & 4.82 & $\begin{array}{c}2.92 \\
(0.86-9.94)\end{array}$ & 0.07 & & \\
\hline Catheter with $>1$ lumen & 6.77 & 2.27 & $\begin{array}{c}2.89 \\
(0.86-9.82)\end{array}$ & 0.07 & & \\
\hline
\end{tabular}

${ }^{*}$ Log-rank test.

** Cox multivariate analysis.

*** ECMO: extracorporeal membrane oxygenation; HR: hazard ratio; DVT: deep vein thrombosis; CI: confidence interval. 
analysis, only a history of cardiovascular surgery and the use of catheters other than peripherally inserted ones were independent risk factors for thrombosis, with a HR of 3.8 (95\% CI: 1.6-9) and of 2.75 (95\% CI: 1.17-6.45), respectively (Table 2, Figure 3).

\section{DISCUSSION}

At the NICU, CVCs are essential for a safe fluid and drug administration in critically ill patients. ${ }^{3,4}$ Nevertheless, their use entails some risks: it may result in mechanical, infectious, and thrombotic complications. ${ }^{5}$ Among newborn infants, more than $90 \%$ of thrombi are related to CVC use. ${ }^{6,7}$

The reported incidence of neonatal CVCrelated thrombosis varies greatly and depends on patients' characteristics, the inserted catheter, and diagnostic tests used. ${ }^{8-10}$ Thrombosis incidence rates ranging from 0.8 to $14.9 \%$ and incidence density rates from 0.4 to $13.2 \%$ catheter-days have been reported. ${ }^{11-13}$ The incidence rates observed in this study when considering only the onset of clinical signs for thrombosis diagnosis were consistent with the lowest values reported in the bibliography; however, this values increased at least 5 times when using a systematic screening protocol based on imaging tests.

Thrombosis development among newborn infants results from a combination of risk factors. ${ }^{14-17}$ The most important one is the presence of a CVC. ${ }^{18,19}$ Catheters may favor the formation of thrombi in newborn infants through damage to the vessel wall, disruption of blood flow in small-diameter vessels, type of catheter used, and infusion of hyperosmolar substances, among other mechanisms. ${ }^{3}$

Dubbink-Verheij et al., ${ }^{12}$ reported a lower incidence of thrombosis when using umbilical and peripherally inserted catheters compared to femoral catheters in newborn infants with a gestational age $\geq 34$ weeks. In a systematic review and metaanalysis on CVC-related venous thrombosis in children, Vidal et al., ${ }^{20}$ also observed a lower frequency of thrombosis associated with the use of umbilical and peripherally inserted catheters. Dhir et al. ${ }^{21}$ described a lower risk for thrombosis when using peripherally inserted catheters compared to long-term CVCs in children.

Similarly, our study found that the use of catheters other than peripherally inserted ones was associated with a higher risk for developing this complication. Therefore, when a newborn infant requires a CVC, peripherally inserted catheters seem to be the safest option.

The second independent risk factor observed in our study for catheter-related thrombosis was a history of cardiovascular surgery.

Pediatric patients who undergo heart surgery have a higher risk of developing thrombosis when compared to other hospitalized children. ${ }^{22,23}$ There are several risk factors that have been associated with the development of thrombosis among these patients: young age (surgery in the neonatal period, ${ }^{22,25}$ infants younger than 1 year) ${ }_{,}{ }^{26}$ oxygen saturation $<85 \%,{ }^{22,25}$ use of deep hypothermic circulatory arrest, ${ }^{22}$ transfusion of blood products during surgery ${ }^{27,28}$ or after cardiopulmonary bypass ${ }^{25}$ postoperative use of extracorporeal support, ${ }^{22}$ CVC use ${ }^{26}$ (indwelling time, ${ }^{22,24}$ presence of more than 1 catheter), ${ }^{24}$ among others.

Most CVC-related thrombi are asymptomatic. ${ }^{29}$ In our cohort, only 3 events had clinical manifestations: superior vena cava syndrome or local symptoms in the lower limbs. This finding proves the need for detection protocols that include imaging tests, given the low frequency of thrombosis diagnosed using clinical manifestations as the only screening method.

This study has certain limitations. On the one side, during the study period, 22 cases of DVT were confirmed; therefore, the number of events was not powerful enough to allow the detection of less common risk factors. On the other side, our unit has a reduced percentage of local births: most patients are referred from other hospitals, so there was a small number of umbilical catheters in our cohort.

Finally, knowing the risk factors associated with the development of thrombosis allows to create a clinical and ultrasound protocol of active surveillance at the NICU in order to screen for and treat thrombotic complications in a timely manner. Larger studies are required to support our findings and identify additional risk factors associated with the development of this complication.

\section{CONCLUSIONS}

The incidence of catheter-related DVT was 5.33 per 1000 catheter-days. Most events occurred in the first 15 days of follow-up. A history of cardiovascular surgical procedures and the use of central catheters others than peripherally inserted ones, were associated with a higher risk of this complication. 


\section{Acknowledgments}

The authors would like to thank Fundación Hospital Garrahan for funding the grant to conduct this study.

\section{REFERENCES}

1. Kenet GNowak-Götti U. Venous thromboembolism in neonates and children. Best Pract Res Clin Hematol. 2012; 25(3):333-44.

2. Nowak-Göttl U, Janssen V, Manner D, Kenet G. Venous thromboembolism in neonates and children. Update 2013. Thromb Res. 2013; 131(Suppl 1):S39-41.

3. Van Ommen CH, Sol JJ. Developmental Hemostasis and Management of Central Venous Catheter Thrombosis in Neonates. Semin Thromb Hemost. 2016; 42(7):752-9.

4. Haddad H, Lee K, Higgins A, McMillan D, et al. Routine Surveillance Ultrasound for the Management of Central Venous Catheters in Neonates. J Pediatr. 2014; 164(1):118- 22.

5. Hermansen MC, Hermansen MG. Intravascular catheter complications in the neonatal intensive care unit. Clin Perinatol. 2005; 32(1):141-56.

6. Andrew M, David M, Adams M, Anderson R, et al. Venous thromboembolic complications (VTE) in children: first analyses of the Canadian Registry of VTE. Blood. 1994; 83(5):1251-7.

7. Van Ommen $\mathrm{CH}$, Heijboer $\mathrm{H}$, Van den Dool EJ, Hutten $\mathrm{BA}$, et al. Pediatric venous thromboembolic disease in one single center: congenital prothrombotic disorders and the clinical outcome. J Thromb Haemost. 2003; 1(12):2516-22.

8. Sol JJ, Van de Loo M, Boerma M, Bergman $K$, et al. NEOnatal Central venous Line Observational study on Thrombosis (NEOCLOT): evaluation of a national guideline on management of neonatal catheter-related thrombosis. BMC Pediatr. 2018; 18(1):84.

9. Park CK, Paes BA, Nagel K, Chan A, et al. Neonatal central venous catheter thrombosis: diagnosis, management and outcome. Blood Coagul Fibrinolysis. 2014; 25(2):97-106.

10. Van Elteren HA, Veldt HS, Te Pas AB, Roest AA, et al. Management and outcome in 32 neonates with thrombotic event. Int J Pediatr. 2011; 2011:217564.

11. Tsai MH, Lien R, Wang JW, Huang HR, et al. Complication Rates With Central Venous Catheters Inserted at Femoral and Non-Femoral Sites in Very Low Birth Weight Infants. Pediatr Infect Dis J. 2009; 28(11):966-70.

12. Dubbink-VerheijGH, Pelsma ICM, Van Ommen CH,SmitsWintjens VE, et al. Femoral Vein Catheterism Important Risk Factor for Catheter-related Thrombosis in (Near-) term Neonates. J Pediatr Hematol Oncol. 2018; 40(2):e64-8.

13. Thornburg CD, Smith PB, Smithwick ML, Cotton CM, et al. Association between thrombosis and blood stream infection in neonates with peripherally inserted catheters. Thromb Res. 2008; 122(6):782-5.

14. HaleyK. Neonatal Venous Thromboembolism. FrontPediatr. 2017; 5:136.
15. Makatsariya A, Bitsadze V, Khizroeva J, Khizroeva J, et al. Neonatal Thrombosis. J Matern Fetal Neonatal Med. 2020; 23:1-9.

16. Bhat R, Kumar R, Kwon S, Murthy K, et al. Risk Factors for Neonatal Venous and Arterial Thromboembolism in the Neonatal Intensive Care Unit- A Case Control Study. J Pediatr. 2018; 195:28-32.

17. Rühle F, Stoll M. Advances in predicting venous thromboembolism risk in children. Br J Haematol. 2018; 180(5):654-65.

18. Amankwah EK, Atchison CM, Arlikar S, Ayala I, et al. Risk factors for hospital-associated venous thromboembolism in the neonatal intensive care unit. Thromb Res. 2014; 134(2):305-9.

19. Andrew M, Monagle P, Brooker LA. Thromboembolic Complications During Infancy and Childhood. Hamilton, London: B.C. Decker; 2000.

20. Vidal E, Sharathkumar A, Glover J, Faustino EV. Central venous catheter-related thrombosis and thromboprophylaxis in children: a systematic review and meta-analysis. J Thromb Haemost. 2014; 12(7):1096-109.

21. Dhir A, DeMarsh S, Ramgopal A, Worley S, et al. Central Venous Line Associated Deep Vein Thrombosis in Hospitalized Children. J Pediatr Hematol Oncol. 2019; 41(7):e432-7.

22. Manlhiot C, Menjak IB, Brandão LR, Gruenwald CE, et al. Risk, clinical features, and outcomes of thrombosis associated with pediatric cardiac surgery. Circulation. 2011; 124(4):1511-9.

23. Guzzetta NA. Thrombosis in Neonates and Infants After Cardiac Surgery-Another Piece of the Puzzle. JCardiothorac Vasc Anesth. 2017; 31(6):1949-51.

24. Steen EH, Lasa JJ, Nguyen TC, Keswani SG, et al. Central Venous Catheter-Related Deep Vein Thrombosis in the Pediatric Cardiac Intensive Care Unit. J Surg Res. 2019; 241:149-59.

25. Ali U, Goldenberg N, Foreman C, Lynn LC, etal. Association Between Cyanosis, Transfusion, and Thrombotic Complications in Neonates and Children Undergoing CardiacSurgery. J Cardiothorac Vasc Anesth. 2020;34(2):34955.

26. Atchison C, Amankwah E, Wilhelm J, Arlikar S, et al. Risk factors for hospital-associated venous thromboembolism in critically ill children following cardiothoracic surgery or therapeutic cardiac catheterization. Cardiol Young. 2018; 28(2):234-42.

27. Faraoni D, Emani S, Halpin E, Bernier R, et al. Relationship Between Transfusion of Blood Products and the Incidence of Thrombotic Complications in Neonates and Infants Undergoing Cardiac Surgery. J Cardiothorac Vasc Anesth. 2017; 31(6):1943-8.

28. Murphy LD, Benneyworth BD, Moser EAS, Hege KM, et al. Analysis of Patient Characteristics and Risk Factors for Thrombosis After Surgery for Congenital Heart Disease. Pediatr Crit Care Med. 2018; 19(12):1146-52.

29. Saxonhouse MA. Management of neonatal thrombosis. Clin Perinatol. 2012; 39(1):191-208. 